hep-th/9412177

\title{
THE ONE-LOOP DIVERGENCES OF
}

\section{THE LINEAR GRAVITY WITH THE TORSION TERMS}

\author{
M. Yu. Kalmykov日 \\ Bogoliubov Laboratory of Theoretical Physics, Joint Institute for Nuclear Research, \\ 141980 Dubna (Moscow Region), Russian Federation \\ and \\ P. I. Pronin'f \\ Department of Theoretical Physics, Physics Faculty \\ Moscow State University, 117234, Moscow, Russian Federation
}

\begin{abstract}
We investigate the role of the torsion field at the quantum level. One-loop counterterms are calculated in the theory with terms quadratic in the torsion field. We have shown that the theory is finite at the one-loop level.
\end{abstract}

\footnotetext{
${ }^{1}$ E-mail: kalmykov@thsun1.jinr.dubna.su

Supported in part by RFFR grant \# 94-02 03665-a and ISF grant \# RFL300

${ }^{2}$ E-mail: pronin@theor.phys.msu.su
} 


\section{Introduction}

The construction of a quantum theory of gravity is an unresolved problem of modern theoretical physics. It is well know that the Einstein theory of gravity is not renormalizable in an ordinary sense [1, 2, 3]. Therefore, one needs to modify the theory or to show that the difficulties presently encountered in the theory are only artifacts of perturbation theory. The simplest method of modifying the Einstein theory is to introduce terms quadratic in the curvature tensor in the action of the theory. This theory is renormalizable and asymptotically free but it is not unitary because the ghosts and tachyons are present in the spectrum of the theory [4, 5]. It should be noted that the unitarity of the theory cannot be restored by means of loop corrections or adding an interaction with matter fields [6, 7]. Hence, one needs to use a new method in order to construct a theory of gravity.

Among various methods of constructing a quantum theory of gravity one should emphasize the gauge approach as the most promising [8, 9, 10, 11]. In the gauge treatment of gravity there are two sets of dynamical variables, namely, the vierbein $h^{a}{ }_{\mu}(x)$ and local Lorentz connection $\omega_{b \mu}^{a}(x)$ or metric $g_{\mu \nu}(x)$ and affine connection $\Gamma_{\mu \nu}^{\sigma}(x)$. The theory based on the first set of variables is called the Poincaré gauge gravitational theory with the structure group $P_{10}$ [12]. A curvature tensor $R_{b \mu \nu}^{a}(\omega)$ and a torsion tensor $Q_{\mu \nu}^{a}(h, \omega)$, which are the strength tensors of the Poincaré gauge gravitational theory, are defined by the following relations:

$$
\begin{aligned}
R_{b \mu \nu}^{a}(\omega) & =\partial_{\mu} \omega_{b \nu}^{a}-\partial_{\nu} \omega_{b \mu}^{a}+\omega_{c \mu}^{a} \omega_{b \nu}^{c}-\omega^{a}{ }_{c \nu} \omega_{b \mu}^{c} \\
Q_{\mu \nu}^{a}(h, \omega) & =-\frac{1}{2}\left(\partial_{\mu} h_{\nu}^{a}-\partial_{\nu} h_{\mu}^{a}+\omega^{a}{ }_{c \nu} h_{\mu}^{c}-\omega_{c \mu}^{a} h_{\nu}^{c}\right)
\end{aligned}
$$

The theory based on the second set of variables is called the affine gauge gravitational theory with the structure gauge group $G A(4, R)$ [13, 14. The strength tensor of the theory is the curvature tensor $\tilde{R}_{\lambda \mu \nu}^{\sigma}(\Gamma)$ defined as:

$$
\tilde{R}_{\lambda \mu \nu}^{\sigma}(\Gamma)=\partial_{\mu} \Gamma_{\lambda \nu}^{\sigma}-\partial_{\nu} \Gamma_{\lambda \mu}^{\sigma}+\Gamma_{\alpha \mu}^{\sigma} \Gamma_{\lambda \nu}^{\alpha}-\Gamma_{\alpha \nu}^{\sigma} \Gamma_{\lambda \mu}^{\alpha}
$$

The Lagrangian of a gauge theory is built out of terms quadratic in the strength tensor of fields. In the Poincaré or affine gauge theories the Lagrangians are defined as

$$
\begin{aligned}
L_{P_{10}} & =\left(\frac{A_{i}}{k^{2}} Q^{2}(h, \omega)+B_{j} R^{2}(\omega)\right) h \\
L_{G A(4, R)} & =C_{j} \tilde{R}^{2}(\Gamma) \sqrt{-g}
\end{aligned}
$$

where $A_{i}, B_{j}$ and $C_{j}$ are arbitrary constants, and $R^{2}$ and $Q^{2}$ are now a symbolic notation for the contractions of the curvature or the torsion tensors, respectively.

For the classical limit, coinciding with the Einstein theory, to exist one needs to add a term linear in the curvature tensor to the Lagrangian.

At the present time, there are a lot of papers concerning the classical problems of the Poincaré and affine gauge gravitational theories [15, 16, 17, 18, 19. However, the 
renormalizability properties of the theories have been insufficiently studied [20, 21, 22, 23, 24.

In the above-mentioned theories, the torsion tensor has a different meaning. In the Poincaré gauge gravitational theory, the torsion tensor is a strength tensor of the tetrad fields. Hence, the terms quadratic in the torsion field must be present in the Lagrangian of the theory. In the affine gauge theory of gravity, the torsion tensor plays an auxiliary role. Therefore, the Lagrangian of the affine-metric theory of gravity can not contain terms quadratic in the torsion field.

The main goal of our work is to investigate the role of the torsion field in the affinemetric theory of gravity at the quantum level. The consideration of the full affine gauge theory described by the action (2) is very cumbersome and technically complex. To understand the role of the torsion fields in the affine-metric theory we will consider a simple model with the terms quadratic in the torsion fields. The Lagrangian of the model is the following:

$$
S_{g r}=-\frac{1}{k^{2}} \int d^{4} x \sqrt{-g}\left(\tilde{R}(\Gamma)-2 \Lambda+b_{1} Q_{\sigma \mu \nu} Q^{\sigma \mu \nu}+b_{2} Q_{\sigma \mu \nu} Q^{\nu \sigma \mu}+b_{3} Q_{\sigma} Q^{\sigma}\right)
$$

where $\Lambda$ is a cosmological constant and $\left\{b_{i}\right\}$ are arbitrary constants.

We consider $\Gamma_{\mu \nu}^{\sigma}(x)$ and $g_{\mu \nu}(x)$ as independent dynamical fields.

This model is not an affine gauge theory in the above-mentioned sense. However, some properties of the affine metric theory can be studied by means of the model (3). In particular, this model possesses the same symmetries as the affine gauge theory.

The main obstacle to the renormalizability of Einstein's theory consists in the existence of the dimensional constant (Newton's constant) and thus in the need for new counterterms in each order of perturbation theory. In other words, renormalizable quantum gravity in four-dimensional space-time must contain terms with dimension four. However, the presence of an additional symmetry in the theory may improve the renormalization properties of the theory. For example, because of the presence of supersymmetry the terms violating the renormalizability of supergravity show up only in higher loops. The considered model (3) has the Lagrangian of dimension two. Hence, this theory is not renormalizable in all orders of perturbation theory. However, the projective invariance [25, 26] existing in the model with metric and affine connection as independent dynamical variables may influence the renormalizability of the theory 27.

In the present work we will research the following problems:

1. The role of the terms quadratic in the torsion fields at the quantum level in the theory with independent metric and affine fields.

2. The influence of an additional projective symmetry on the one-loop counterterms.

3. The influence of the terms quadratic in the torsion fields on the one-loop renormalizability of the theory. 
We use the following notation:

$$
\begin{array}{ccc}
c=\hbar=1 ; \quad \mu, \nu=0,1,2,3 ; \quad k^{2}=16 \pi G & \varepsilon=\frac{4-n}{2} \\
\tilde{R}_{\mu \nu}(\Gamma)=\tilde{R}_{\mu \sigma \nu}^{\sigma}(\Gamma), \quad \tilde{R}(\Gamma)=\tilde{R}_{\mu \nu}(\Gamma) g^{\mu \nu}, \quad g=\operatorname{det}\left(g_{\mu \nu}\right)
\end{array}
$$

The objects marked by the tilde $\sim$ are constructed by means of the affine connection $\Gamma_{\mu \nu}^{\sigma}$. The others are the Riemannian objects.

\section{Symmetries of the model and equations of motion}

Let us consider the classical symmetries of the model (3). This model is invariant under the general coordinate transformation

$$
\begin{aligned}
x^{\mu} & \rightarrow{ }^{\prime} x^{\mu}=x^{\mu}+k \xi^{\mu}(x) \\
g_{\mu \nu}(x) & \rightarrow{ }^{\prime} g_{\mu \nu}(x)=g_{\mu \nu}(x)-k \partial_{\mu} \xi^{\alpha} g_{\alpha \nu}(x)-k \partial_{\nu} \xi^{\alpha} g_{\alpha \mu}(x)-k \xi^{\alpha} \partial_{\alpha} g_{\mu \nu}(x)+O\left(k^{2}\right) \\
\Gamma_{\mu \nu}^{\sigma}(x) \rightarrow{ }^{\prime} \Gamma_{\mu \nu}^{\sigma}(x) & =\Gamma_{\mu \nu}^{\sigma}(x)-k \partial_{\mu} \xi^{\alpha} \Gamma_{\alpha \nu}^{\sigma}(x)-k \partial_{\nu} \xi^{\alpha} \Gamma_{\mu \alpha}^{\sigma}(x) \\
& \quad+k \partial_{\alpha} \xi^{\sigma} \Gamma_{\mu \nu}^{\alpha}(x)-k \xi^{\alpha} \partial_{\alpha} \Gamma_{\mu \nu}^{\sigma}(x)-k \partial_{\mu \nu} \xi^{\sigma}+O\left(k^{2}\right)
\end{aligned}
$$

Moreover, in the case of special choice of the coefficients $\left\{b_{j}\right\}$, the action (3) is invariant under the following transformation of fields:

$$
\begin{aligned}
x^{\mu} & \rightarrow{ }^{\prime} x^{\mu}=x^{\mu} \\
g_{\mu \nu}(x) & \rightarrow{ }^{\prime} g_{\mu \nu}(x)=g_{\mu \nu}(x) \\
\Phi_{m a t}(x) & \rightarrow{ }^{\prime} \Phi_{m a t}(x)=\Phi_{m a t}(x) \\
\Gamma_{\mu \nu}^{\sigma}(x) & \rightarrow{ }^{\prime} \Gamma_{\mu \nu}^{\sigma}(x)=\Gamma_{\mu \nu}^{\sigma}(x)+k \delta_{\mu}^{\sigma} C_{\nu}(x)
\end{aligned}
$$

where $C_{\nu}(x)$ is an arbitrary vector.

This is the projective transformation [25], 26].

It is easy to show that under the projective transformations (5) the curvature and torsion tensors transform in the following way:

$$
\begin{aligned}
\tilde{R}_{\lambda \mu \nu}^{\sigma}(\Gamma) & \rightarrow{ }^{\prime} \tilde{R}_{\lambda \mu \nu}^{\sigma}\left({ }^{\prime} \Gamma\right)=\tilde{R}_{\lambda \mu \nu}^{\sigma}(\Gamma)+\delta_{\lambda}^{\sigma} k\left(\partial_{\mu} C_{\nu}-\partial_{\nu} C_{\mu}\right) \\
Q_{\mu \nu}^{\sigma} & \rightarrow{ }^{\prime} Q_{\mu \nu}^{\sigma}=Q_{\mu \nu}^{\sigma}+\frac{1}{2} k\left(\delta_{\mu}^{\sigma} C_{\nu}-\delta_{\nu}^{\sigma} C_{\mu}\right)
\end{aligned}
$$

Hence, the action (3) is invariant under the projective transformation (5) at the tree level only under the condition

$$
2 b_{1}+3 b_{3}-b_{2}=0
$$

The classical fields ${ }_{\mu \nu}$ and $g_{\mu \nu}$ satisfy the following equations of motion: 


$$
\begin{aligned}
\frac{\delta S_{g r}}{\delta g^{\mu \nu}} & =\tilde{R}_{(\mu \nu)}-\frac{1}{2} \tilde{R} g_{\mu \nu}+\Lambda g_{\mu \nu} \\
& -b_{1} Q_{\mu \alpha \beta} Q_{\nu}^{\alpha \beta}+2 b_{1} Q_{\alpha \beta \mu} Q_{\nu}^{\alpha \beta}-b_{2} Q_{\mu}^{\alpha b} Q_{\beta \alpha \nu}+b_{3} Q_{\mu} Q_{\nu} \\
& +\frac{1}{2} g_{\mu \nu}\left(b_{1} Q_{\sigma \alpha \beta} Q^{\sigma \alpha \beta}+b_{2} Q_{\sigma \alpha \beta} Q^{\beta \sigma \alpha}+b_{3} Q_{\sigma} Q^{\sigma}\right)=0
\end{aligned}
$$

and

$$
\begin{aligned}
\frac{\delta S_{g r}}{\delta \Gamma_{\alpha \beta}^{\sigma}} & =D_{\lambda}^{\alpha \lambda} \delta_{\sigma}^{\beta}+D_{\sigma} g^{\alpha \beta}-\left(1-\frac{1}{2} b_{2}\right) D_{\sigma}^{\alpha \beta}-\left(1-\frac{1}{2} b_{2}\right) D_{\sigma}^{\beta \alpha} \\
& +b_{1} D_{\sigma}^{\alpha \beta}-b_{1} D_{\sigma}^{\beta \alpha}-\frac{1}{2} b_{2} D_{\sigma}^{\alpha \beta}-\frac{1}{2} b_{2} D_{\sigma}^{\beta \alpha} \\
& +\frac{1}{2} b_{3} D^{\alpha} \delta_{\sigma}^{\beta}-\frac{1}{2} b_{3} D^{\beta} \delta_{\sigma}^{\alpha}-\frac{1}{2} b_{3} D_{\lambda}^{\lambda \alpha} \delta_{\sigma}^{\beta}+\frac{1}{2} b_{3} D_{\lambda}^{\lambda \beta} \delta_{\sigma}^{\alpha}=0
\end{aligned}
$$

where

$$
\begin{aligned}
D_{\mu \nu}^{\sigma} & =\Gamma_{\mu \nu}^{\sigma}-g^{\sigma \lambda} \frac{1}{2}\left(-\partial_{\lambda} g_{\mu \nu}+\partial_{\mu} g_{\nu \lambda}+\partial_{\nu} g_{\mu \lambda}\right) \\
D_{\sigma} & =D_{\sigma \alpha}^{\alpha}
\end{aligned}
$$

Equation ( 8) has two solutions

1. if $2 b_{1}+3 b_{3}-b_{2}=0$,

$$
D_{\mu \nu}^{\sigma}=\delta_{\mu}^{\sigma} C_{\nu}(x)
$$

where $C_{\nu}$ is an arbitrary vector.

2. if $2 b_{1}+3 b_{3}-b_{2} \neq 0$

$$
D_{\mu \nu}^{\sigma}=0
$$

Taking into account (9) or (10) we obtain from (7)

$$
R_{\mu \nu}=\Lambda g_{\mu \nu}
$$

In the next chapter, we will consider at the quantum level two cases:

- the theory without the projective invariance (the condition (6) is not satisfied.)

- the theory with the projective invariance (the condition (6) is fulfilled) 


\section{One-loop counterterms}

For calculating the one-loop effective action we will use the background field method [28, 29] and the Schwinger-DeWitt technique [30, 31]. In the gauge theories, the renormalization procedure may violate the gauge invariance at the quantum level, thus destroying the renormalizability of the theory. Therefore, one is bound to apply an invariant renormalization. We will use the dimensional regularization and minimal subtraction scheme in our loop calculation. This is the invariant renormalization.

In accordance with the background field method, all dynamical variables are rewritten as a sum of classical and quantum parts. In general case, the dynamical variables in the affine-metric theory are $\underline{\Gamma}_{\mu \nu}^{\sigma}, \bar{g}_{\mu \nu}=g_{\mu \nu}(-g)^{r}$ or $\bar{g}^{\mu \nu}=g^{\mu \nu}(-g)^{s}$, where $r, s$ are the numbers satisfying the only condition: $r \neq-\frac{1}{4}, s \neq \frac{1}{4}$. The one-loop counterterms on the mass-shell do not depend on the value of $r$ and $s$. To simplify our calculation, we use the following numbers $r=s=0$.

The fields $\underline{\Gamma}_{\mu \nu}^{\sigma}$ and $\underline{g}_{\mu \nu}$ are now rewritten according to

$$
\begin{aligned}
\underline{\Gamma}_{\mu \nu}^{\sigma} & =\Gamma_{\mu \nu}^{\sigma}+k \gamma_{\mu \nu}^{\sigma} \\
\underline{g}_{\mu \nu} & =g_{\mu \nu}+k h_{\mu \nu}
\end{aligned}
$$

where $\Gamma_{\mu \nu}^{\sigma}, g_{\mu \nu}$ are the classical parts satisfying equations (7) and (8).

The action (3) expanded as a power series in the quantum fields (12) defines the effective action for calculating the loop counterterms. The one-loop effective Lagrangian quadratic in the quantum fields is

$$
L_{e f f}=-\frac{1}{2} \gamma_{\mu \nu}^{\sigma} \tilde{F}_{\sigma \lambda}^{\mu \nu \alpha \beta} \gamma_{\alpha \beta}^{\lambda}-\frac{1}{2} h^{\alpha \beta} X_{\alpha \beta \mu \nu} h^{\mu \nu}-\gamma_{\alpha \beta}^{\lambda} B_{\lambda}^{\alpha \beta \sigma}{ }_{\mu \nu} \nabla_{\sigma} h^{\mu \nu}+\gamma_{\mu \nu}^{\sigma} H_{\sigma}^{\mu \nu}{ }_{\alpha \beta} h^{\alpha \beta}
$$

where

$$
\begin{aligned}
\tilde{F}_{\alpha \mu}^{\beta \lambda \nu \sigma} & =g^{\beta \lambda} \delta_{\alpha}^{\nu} \delta_{\mu}^{\sigma}+g^{\nu \sigma} \delta_{\alpha}^{\lambda} \delta_{\mu}^{\beta}-\left(1-\frac{b_{2}}{2}\right) g^{\beta \sigma} \delta_{\alpha}^{\nu} \delta_{\mu}^{\lambda}-\left(1-\frac{b_{2}}{2}\right) g^{\lambda \nu} \delta_{\alpha}^{\sigma} \delta_{\mu}^{\beta} \\
& +b_{1} g_{\alpha \mu} g^{\beta \nu} g^{\sigma \lambda}-b_{1} g_{\alpha \mu} g^{\lambda \nu} g^{\beta \sigma}-\frac{b_{2}}{2} g^{\lambda \sigma} \delta_{\mu}^{\beta} \delta_{\alpha}^{\nu}-\frac{b_{2}}{2} g^{\beta \nu} \delta_{\mu}^{\lambda} \delta_{\alpha}^{\sigma} \\
& +\frac{b_{3}}{2} g^{\beta \nu} \delta_{\alpha}^{\lambda} \delta_{\mu}^{\sigma}-\frac{b_{3}}{2} g^{\beta \sigma} \delta_{\mu}^{\nu} \delta_{\alpha}^{\lambda}-\frac{b_{3}}{2} g^{\lambda \nu} \delta_{\alpha}^{\beta} \delta_{\mu}^{\sigma}+\frac{b_{3}}{2} g^{\sigma \lambda} \delta_{\alpha}^{\beta} \delta_{\mu}^{\nu} \\
P^{\alpha \beta \mu \nu}= & \frac{1}{4}\left(g^{\alpha \mu} g^{\beta \nu}+g^{\alpha \nu} g^{\beta \mu}-g^{\alpha \beta} g^{\mu \nu}\right) \\
B_{\lambda}^{\alpha \beta \sigma}(\mu \nu)= & 2\left(\delta_{\lambda}^{\sigma} P_{\mu \nu}^{\alpha \beta}-\delta_{\lambda}^{\beta} P_{\mu \nu}^{\alpha \sigma}\right) \\
X_{((\alpha \beta)(\mu \nu))}= & 2 R_{\alpha \mu} g_{\beta \nu}-(R-2 \Lambda) P_{\alpha \beta \mu \nu}-R_{\alpha \beta} g_{\mu \nu}-\frac{1}{2} b_{1} g_{\alpha \beta} Q_{\mu \sigma \lambda} Q_{\nu}^{\sigma \lambda} \\
+ & b_{1}\left(2 Q_{\alpha \mu}^{\sigma} Q_{\sigma \beta \nu}-4 Q_{\alpha \mu \sigma} Q_{\beta \nu}^{\sigma}+4 Q_{\alpha}^{\sigma \lambda} Q_{\sigma \lambda \mu} g_{\beta \nu}-g_{\alpha \beta} Q_{\mu}^{\sigma \lambda} Q_{\sigma \lambda \nu}\right)
\end{aligned}
$$




$$
\begin{aligned}
& +b_{2}\left(2 g_{\beta \mu} Q_{\alpha}^{\sigma \lambda} Q_{\lambda \nu \sigma}-g_{\alpha \beta} Q_{\mu}^{\sigma \lambda} Q_{\lambda \nu \sigma}\right)+b_{3}\left(g_{\alpha \beta} Q_{\mu} Q_{\nu}-2 g_{\alpha \mu} Q_{\beta} Q_{\nu}\right) \\
& -P_{\alpha \beta \mu \nu}\left(b_{1} Q^{\lambda \rho \tau} Q_{\lambda \rho \tau}+b_{2} Q_{\lambda \rho \tau} Q^{\tau \lambda \rho}+b_{3} Q^{\lambda} Q_{\lambda}\right) \\
H_{\sigma}^{\mu \nu}(\alpha \beta) & =2\left(D_{\rho \tau}^{\mu} \delta_{\sigma}^{\nu}+D_{\sigma} \delta_{\rho}^{\mu} \delta_{\tau}^{\nu}-D_{\rho \sigma}^{\mu} \delta_{\tau}^{\nu}-D_{\sigma \tau}^{\nu} \delta_{\rho}^{\mu}\right) P_{\alpha \beta}^{\rho \tau} \\
& +2 b_{1}\left(Q_{\sigma \alpha}^{\mu} \delta_{\beta}^{\nu}-Q_{\sigma \alpha}^{\nu} \delta_{\beta}^{\mu}-Q_{\alpha}^{\mu \nu} g_{\beta \sigma}-\frac{1}{2} Q_{\sigma}^{\mu \nu} g_{\alpha \beta}\right) \\
& +2 b_{2}\left(Q_{\sigma \lambda}^{\nu} P_{\alpha \beta}^{\mu \lambda}-Q_{\sigma \lambda}^{\mu} P_{\alpha \beta}^{\nu \lambda}\right)+2 b_{3}\left(\delta_{\sigma}^{\nu} Q_{\lambda} P_{\alpha \beta}^{\mu \lambda}-\delta_{\sigma}^{\mu} Q_{\lambda} P_{\alpha \beta}^{\nu \lambda}\right)
\end{aligned}
$$

Parentheses around index pairs denote symmetrization while parentheses around four indices mean symmetrization also under pair interchange. These symmetries are automatically enforced by the symmetries of the quantum fields multiplying these quantities.

Let us consider the first case: the theory without the projective invariance (the condition (6) is not satisfied). To get the diagonal form of the effective Lagrangian we are to replace the dynamical variables in the following way:

$$
\tilde{\gamma}_{\mu \nu}^{\sigma}=\gamma_{\mu \nu}^{\sigma}+F_{\mu \nu \alpha \beta}^{-1 \sigma \lambda}\left(B_{\lambda}^{\alpha \beta \tau}{ }_{\rho \epsilon} \nabla_{\tau}-H_{\lambda}^{\alpha \beta}{ }_{\rho \epsilon}^{\alpha \beta}\right) h^{\rho \epsilon}
$$

where $F_{\mu \nu \alpha \beta}^{-1 \sigma} \lambda_{\alpha \beta}$ is the propagator of the quantum field $\gamma_{\mu \nu}^{\sigma}$ satisfying two conditions

$$
\begin{aligned}
& F_{\mu \nu \alpha \beta}^{-1 \sigma \lambda}=F_{\alpha \beta \mu \nu}^{-1 \lambda}{ }_{\mu \nu} \\
& F_{\mu \nu \alpha \beta}^{-1 \sigma{ }_{\lambda}^{\lambda} \tilde{F}_{\rho}^{\alpha \beta \tau \epsilon}}=\delta_{\rho}^{\sigma} \delta_{\mu}^{\tau} \delta_{\nu}^{\epsilon}
\end{aligned}
$$

Having solved equations (16) and (17) we obtain the following result:

$$
\begin{aligned}
F_{\beta \sigma \nu \lambda}^{-1 \alpha \mu}= & -\frac{1}{4} g^{\alpha \mu} g_{\beta \sigma} g_{\nu \lambda}+A_{2} g^{\alpha \mu} g_{\beta \nu} g_{\sigma \lambda}+\left(\frac{1}{12}-\frac{1}{3}\left(A_{2}-A_{1}\right)\right) g_{\nu \beta} \delta_{\lambda}^{\mu} \delta_{\sigma}^{\alpha} \\
& +\frac{1}{4}\left(g_{\nu \lambda} \delta_{\beta}^{\mu} \delta_{\sigma}^{\alpha}+g_{\beta \sigma} \delta_{\nu}^{\alpha} \delta_{\lambda}^{\mu}\right)+A_{1}\left(g_{\nu \sigma} \delta_{\lambda}^{\alpha} \delta_{\beta}^{\mu}+g_{\beta \lambda} \delta_{\sigma}^{\mu} \delta_{\nu}^{\alpha}\right) \\
& +\frac{1}{4}\left(g_{\nu \lambda} \delta_{\sigma}^{\mu} \delta_{\beta}^{\alpha}+g_{\beta \sigma} \delta_{\lambda}^{\alpha} \delta_{\nu}^{\mu}\right)-\left(A_{2}-\frac{1}{2}\right) g^{\alpha \mu} g_{\sigma \nu} g_{\beta \lambda} \\
& +\left(\frac{1}{3}\left(A_{2}-A_{1}\right)-\frac{1}{4}\right)\left(g_{\beta \lambda} \delta_{\nu}^{\mu} \delta_{\sigma}^{\alpha}+g_{\beta \nu} \delta_{\beta}^{\alpha} \delta_{\lambda}^{\mu}\right) \\
& +\left(\frac{1}{12}-\frac{1}{3}\left(A_{2}-A_{1}\right)+\frac{2}{3\left(2 b_{1}+3 b_{3}-b_{2}\right)}\right) g_{\sigma \lambda} \delta_{\nu}^{\mu} \delta_{\beta}^{\alpha} \\
& -\left(\frac{1}{2}+A_{1}\right) g_{\nu \beta} \delta_{\sigma}^{\mu} \delta_{\lambda}^{\alpha}-\left(\frac{1}{2}+A_{1}\right) g_{\sigma \lambda} \delta_{\beta}^{\mu} \delta_{\nu}^{\alpha}
\end{aligned}
$$

where the constants $A_{2}$ and $A_{1}$ are defined by the following expressions :

$$
\begin{aligned}
& A_{2}=\frac{2 b_{2}^{2}-4 b_{1}^{2}-2 b_{1} b_{2}-6 b_{2}-2 b_{1}+4}{d} \\
& A_{1}=-A_{2}-\frac{4 b_{1}}{d}+\frac{8}{d}
\end{aligned}
$$


where

$$
d \equiv 8\left(b_{2}^{2}-2 b_{2}+1+b_{1}-b_{1} b_{2}-2 b_{1}^{2}\right) \neq 0
$$

The replacement (15) does not change the functional measure

$$
\operatorname{det}\left|\frac{\partial(h, \tilde{\gamma})}{\partial(h, \gamma)}\right|=1
$$

We violate the coordinate invariance of the action (13) by means of the following gauge:

$$
\begin{gathered}
F_{\mu}=\nabla_{\nu} h_{\mu}^{\nu}-\frac{1}{2} \nabla_{\mu} h_{\alpha}^{\alpha} \\
L_{g h}=\frac{1}{2} F_{\mu} F^{\mu}
\end{gathered}
$$

The Lagrangian of the coordinate ghost is

$$
L_{g h}=-\bar{c}^{\mu}\left(g_{\mu \nu} \nabla^{2}+R_{\mu \nu}\right) c^{\nu}
$$

We don't give the details of cumbersome calculations. The one-loop counterterms on the mass-shell including the contributions of the quantum and ghost fields are

$$
\triangle \Gamma_{\infty}^{1}=-\frac{1}{32 \pi^{2} \varepsilon} \int d^{4} x \sqrt{-g}\left(\frac{53}{45} R_{\alpha \beta \mu \nu} R^{\alpha \beta \mu \nu}-\frac{58}{5} \Lambda^{2}\right)
$$

Let us consider the second case: the theory possessing the projective invariance (the condition (6) is fulfilled). In this case, the propagator $F_{\beta \sigma \nu \lambda}^{-1 \alpha}{ }_{\mu}$ of the quantum field $\gamma_{\mu \nu}^{\sigma}$ does not exist because of the projective invariance of the effective Lagrangian (13).

We consider the projective invariance as a gauge symmetry. Hence, we must fix this symmetry at the quantum level. The gauge fixing Lagrangian is

$$
L_{g f}=b^{\mu} F_{\mu}+\pi^{\mu} f_{\mu}-\frac{1}{2} b^{\mu} b_{\mu}-\frac{1}{2} \pi^{\mu} \pi_{\mu}
$$

where $b_{\mu}$ and $\pi_{\mu}$ are additional auxiliary fields. Since they appear without derivatives in the Lagrangian, they can be eliminated by means of their equations of motion which yield

$$
L_{g f}=\frac{1}{2} F_{\mu} F^{\mu}+\frac{1}{2} f_{\mu} f^{\mu}
$$

where $F_{\mu}$ is given in equation (21) and $f_{\mu}$ has the following form [27:

$$
f_{\lambda}=\left(f_{1} g_{\sigma \lambda} g^{\mu \nu}+f_{2} \delta_{\lambda}^{\mu} \delta_{\sigma}^{\nu}+f_{3} \delta_{\lambda}^{\nu} \delta_{\sigma}^{\mu}\right) \gamma_{\mu \nu}^{\sigma}
$$

where $\left\{f_{i}\right\}$ are constants satisfying the condition

$$
f_{1}+f_{2}+4 f_{3} \neq 0
$$


For constructing the quantum Lagrangian we must add the appropriate FaddeevPopov ghost fields. We derive the corresponding theory from the invariance of the full Lagrangian under the BRST-transformation

$$
s L_{\text {quan }}=0
$$

where $s$ is a nilpotent BRST operator. In the background field formalism we violate the symmetry connected with the transformation of quantum fields. The BRSTtransformation is obtained in the usual way from gauge transformation by replacing the gauge parameter by the corresponding ghost field. The complete BRSTtransformations for all fields are the following:

$$
\begin{aligned}
s g_{\mu \nu} & =0 \quad s_{\mu \nu}^{\sigma}=0 \\
s h_{\mu \nu} & =\left(\nabla_{\mu} c_{\nu}+\nabla_{\nu} c_{\mu}\right)+k\left(c^{\lambda} \nabla_{\lambda} h_{\mu \nu}+\nabla_{\mu} c^{\lambda} h_{\lambda \nu}+\nabla_{\nu} c^{\lambda} h_{\lambda \mu}\right)+O\left(k^{2}\right) \\
s c^{\mu} & =c^{\lambda} \partial_{\lambda} c^{\mu} \quad s \bar{c}^{\mu}=b^{\mu} \quad s b^{\mu}=0 \\
s \bar{\chi}^{\mu} & =\pi^{\mu} \quad s \pi^{\mu}=0 \quad s \chi_{\nu}=0 \\
s \gamma_{\mu \nu}^{\sigma}(x) & =D_{\alpha \nu}^{\sigma} \nabla_{\mu} c^{\alpha}+D_{\mu \alpha}^{\sigma} \nabla_{\nu} c^{\alpha}-D_{\mu \nu}^{\alpha} \nabla_{\alpha} c^{\sigma}+c^{\alpha} \nabla_{\alpha} D_{\mu \nu}^{\sigma} \\
& +\frac{1}{2}\left(\nabla_{\mu} \nabla_{\nu}+\nabla_{\nu} \nabla_{\mu}\right) c^{\sigma}-\frac{1}{2}\left(R_{\mu \nu \beta}^{\sigma}+R_{\nu \mu \beta}^{\sigma}\right) c^{\beta}-\delta_{\mu}^{\sigma} \chi_{\nu} \\
& -k\left(\gamma_{\mu \nu}^{\alpha} \nabla_{\alpha} c^{\sigma}-\gamma_{\alpha \nu}^{\sigma} \nabla_{\mu} c^{\alpha}-\gamma_{\mu \alpha}^{\sigma} \nabla_{\nu} c^{\alpha}-c^{\alpha} \nabla_{\alpha} \gamma_{\mu \nu}^{\sigma}\right)+O\left(k^{2}\right)
\end{aligned}
$$

where $\left(\bar{c}_{\mu}, c_{\nu}\right)$ and $\left(\bar{\chi}_{\mu}, \chi_{\nu}\right)$ are the anticommuting ghost fields connected with general coordinate and projective transformations, respectively.

The quantum Lagrangian is

$$
L_{\text {quan }}=L_{\text {eff }}(\gamma, h)+s\left\{\bar{c}^{\mu}\left(F_{\mu}-\frac{1}{2} b_{\mu}\right)+\bar{\chi}^{\mu}\left(f_{\mu}-\frac{1}{2} \pi_{\mu}\right)\right\}
$$

where $L_{\text {eff }}(\gamma, h)$ is the action (3) expanded as a power series in the quantum fields. This, together with the condition $s^{2}=0$, implies immediately the BRST invariance of the action (31).

From (30) and (31) we obtain the one-loop ghost Lagrangian

$$
\begin{aligned}
L_{g h} & =-\bar{c}^{\mu} s F_{\mu}-\bar{\chi}^{\mu} s f_{\mu} \\
& =-\left(\begin{array}{cc}
\bar{c}^{\mu} \bar{\chi}^{\mu}
\end{array}\right)\left(\begin{array}{cc}
\left(g_{\mu \nu} \nabla^{2}+R_{\mu \nu}\right) & 0 \\
Z_{\mu \nu} & \left(f_{1}+f_{2}+4 f_{3}\right) g_{\mu \nu}(-g)^{\alpha}
\end{array}\right)\left(\begin{array}{l}
c^{\nu} \\
\chi^{\nu}
\end{array}\right)
\end{aligned}
$$

where $\alpha$ is a constant and

$$
\begin{aligned}
Z_{\lambda \sigma} & =-f_{1} g_{\lambda \sigma} \nabla^{2}-\frac{1}{2}\left(f_{2}+f_{3}\right)\left(\nabla_{\lambda} \nabla_{\sigma}+\nabla_{\sigma} \nabla_{\lambda}\right)+\frac{1}{2}\left(f_{2}+f_{3}-2 f_{1}\right) R_{\lambda \sigma} \\
& -\left(f_{1} \nabla_{\sigma} D_{\lambda}^{\mu}{ }_{\mu}+f_{2} \nabla_{\sigma} D_{\lambda}+f_{3} \nabla_{\sigma} D_{\mu \lambda}^{\mu}\right)-\left(f_{2} D_{\sigma}+f_{3} D_{\mu \sigma}^{\mu}\right) \nabla_{\lambda} \\
& +f_{1}\left(g_{\lambda \sigma} D^{\alpha \beta}{ }_{\beta} \nabla_{\alpha}-D_{\lambda \sigma}{ }^{\mu} \nabla_{\mu}-D_{\lambda \sigma}^{\mu} \nabla_{\mu}\right)
\end{aligned}
$$


The following relations are valid for arbitrary triangular matrix operator:

$$
\begin{aligned}
\ln \operatorname{det}\left(\begin{array}{ll}
A & 0 \\
C & B
\end{array}\right) & =S p \ln \left(\begin{array}{ll}
A & 0 \\
C & B
\end{array}\right)=S p\left(\begin{array}{cc}
\ln A & 0 \\
K & \ln B
\end{array}\right) \\
& =\ln A+\ln B=\ln \operatorname{det}\left(\begin{array}{cc}
A & 0 \\
0 & B
\end{array}\right)
\end{aligned}
$$

where $\mathrm{A}, \mathrm{B}$ and $\mathrm{C}$ are arbitrary operators.

Using these relations we can write the one-loop ghost contribution to the effective action in a more convenient form

$$
{ }_{g h}=-i \ln \operatorname{det}\left(g_{\mu \nu} \nabla^{2}+R_{\mu \nu}\right)-i \ln \operatorname{det}\left(\left(f_{1}+f_{2}+4 f_{3}\right) g_{\mu \nu}(-g)^{\alpha}\right)
$$

where the first and second terms are the one-loop contribution of the coordinate and projective ghosts, respectively.

The validity of the relation (35) can also be proven in a different way. To get the diagonal form of the ghost Lagrangian (32) we define a new field

$$
\tilde{\chi}_{\nu}=\chi_{\nu}+Z_{\nu \sigma} c^{\sigma}
$$

This redefinition does not change the functional measure. In the new variables the ghost Lagrangian has the diagonal form

$$
L_{g h}-\left(\begin{array}{cc}
\bar{c}^{\mu} \bar{\chi}^{\mu}
\end{array}\right)\left(\begin{array}{cc}
\left(g_{\mu \nu} \nabla^{2}+R_{\mu \nu}\right. & 0 \\
0 & \left(f_{1}+f_{2}+4 f_{3}\right) g_{\mu \nu}(-g)^{\alpha}
\end{array}\right)\left(\begin{array}{c}
c^{\nu} \\
\tilde{\chi}^{\nu}
\end{array}\right)
$$

The ghost contribution to the one-loop effective action is given by the relation (35).

To simplify our calculation we use the following projective gauge condition instead of 27$)$ :

$$
f_{\lambda}=A \delta_{\sigma}^{\beta} \delta_{\lambda}^{\alpha} \gamma_{\alpha \beta}^{\sigma} \equiv f_{\lambda \sigma}^{\alpha \beta} \gamma_{\alpha \beta}^{\sigma}
$$

where the constant $A$ is nonzero.

The one-loop contribution of the projective ghosts to the effective action is proportional to $\delta^{4}(0)$. In the dimensional regularization $\left[\delta^{4}(0)\right]_{R}=0$, and the contribution of the projective ghosts to the one-loop counterterms is equal to zero.

Now, we must change equation (17). The propagator of the quantum field $\gamma_{\mu \nu}^{\sigma}$ satisfies equation (16) and the new condition

$$
\bar{F}_{\mu \nu \alpha \beta}^{-1 \sigma \lambda} \bar{F}_{\lambda \rho}^{\alpha \beta \tau \epsilon}=\delta_{\rho}^{\sigma} \delta_{\mu}^{\tau} \delta_{\nu}^{\epsilon}
$$

where

$$
\begin{aligned}
\bar{F}_{\sigma \lambda}^{\alpha \beta \mu \nu} & =\tilde{F}_{\sigma \lambda}^{\alpha \beta \mu \nu}+f_{\tau \sigma}^{\alpha \beta} f_{\lambda}^{\tau}{ }^{\mu \nu} \\
& =b_{1} g_{\sigma \lambda} g^{\beta \nu} g^{\alpha \mu}-b_{1} g_{\sigma \lambda} g^{\beta \mu} g^{\alpha \nu}-\left(1-\frac{b_{2}}{2}\right) g^{\nu \alpha} \delta_{\sigma}^{\mu} \delta_{\lambda}^{\beta}-\left(1-\frac{b_{2}}{2}\right) g^{\mu \beta} \delta_{\sigma}^{\nu} \delta_{\lambda}^{\alpha}
\end{aligned}
$$




$$
\begin{aligned}
& +\left(A^{2}+\frac{b_{3}}{2}\right) g^{\alpha \mu} \delta_{\lambda}^{\nu} \delta_{\sigma}^{\beta}-\frac{b_{3}}{2} g^{\mu \beta} \delta_{\sigma}^{\alpha} \delta_{\lambda}^{\nu}-\frac{b_{3}}{2} g^{\alpha \nu} \delta_{\sigma}^{\beta} \delta_{\lambda}^{\mu}+\frac{b_{3}}{2} g^{\nu \beta} \delta_{\sigma}^{\alpha} \delta_{\lambda}^{\mu} \\
& +g^{\mu \nu} \delta_{\lambda}^{\alpha} \delta_{\sigma}^{\beta}+g^{\alpha \beta} \delta_{\sigma}^{\mu} \delta_{\lambda}^{\nu}-\frac{b_{2}}{2} g^{\alpha \mu} \delta_{\lambda}^{\beta} \delta_{\sigma}^{\nu}-\frac{b_{2}}{2} g^{\beta \nu} \delta_{\lambda}^{\alpha} \delta_{\sigma}^{\mu}
\end{aligned}
$$

Having solved equations (16) and (39) we obtain the following result:

$$
\begin{aligned}
\bar{F}_{\beta \sigma \nu \lambda}^{-1 \alpha \mu}= & -\frac{1}{4} g^{\alpha \mu} g_{\beta \sigma} g_{\nu \lambda}+A_{2} g^{\alpha \mu} g_{\beta \nu} g_{\sigma \lambda}+\left(\frac{1}{12}-\frac{1}{3}\left(A_{2}-A_{1}\right)\right) g_{\nu \beta} \delta_{\lambda}^{\mu} \delta_{\sigma}^{\alpha} \\
& +\frac{1}{4}\left(g_{\nu \lambda} \delta_{\beta}^{\mu} \delta_{\sigma}^{\alpha}+g_{\beta \sigma} \delta_{\nu}^{\alpha} \delta_{\lambda}^{\mu}\right)+A_{1}\left(g_{\nu \sigma} \delta_{\lambda}^{\alpha} \delta_{\beta}^{\mu}+g_{\beta \lambda} \delta_{\sigma}^{\mu} \delta_{\nu}^{\alpha}\right) \\
& -\frac{1}{4}\left(g_{\nu \lambda} \delta_{\sigma}^{\mu} \delta_{\beta}^{\alpha}+g_{\beta \sigma} \delta_{\lambda}^{\alpha} \delta_{\nu}^{\mu}\right)-\left(A_{2}-\frac{1}{2}\right) g^{\alpha \mu} g_{\sigma \nu} g_{\beta \lambda} \\
& +\left(\frac{1}{3}\left(A_{2}-A_{1}\right)-\frac{1}{12}\right)\left(g_{\beta \lambda} \delta_{\nu}^{\mu} \delta_{\sigma}^{\alpha}+g_{\beta \nu} \delta_{\beta}^{\alpha} \delta_{\lambda}^{\mu}\right) \\
& +\left(\frac{1}{12}-\frac{1}{3}\left(A_{2}-A_{1}\right)+A^{2}\right) g_{\sigma \lambda} \delta_{\nu}^{\mu} \delta_{\beta}^{\alpha} \\
& -\left(\frac{1}{2}+A_{1}\right) g_{\nu \beta} \delta_{\sigma}^{\mu} \delta_{\lambda}^{\alpha}-\left(\frac{1}{2}+A_{1}\right) g_{\sigma \lambda} \delta_{\beta}^{\mu} \delta_{\nu}^{\alpha}
\end{aligned}
$$

where the constants $A_{2}, A_{1}$ are defined from expressions (19).

The abandonment calculations coincide with the previous case. Having made the replacement of the variables (15), one needs to change $F_{\beta \sigma \nu \lambda}^{-1 \alpha_{\mu}^{\mu}} \rightarrow \bar{F}_{\beta \sigma \nu \lambda}^{-1 \alpha}{ }_{\beta \sigma}$. We fix the coordinate invariance by the conditions (21) and (22), and the Lagrangian of the coordinate ghosts is defined by (23). The one-loop counterterms on mass-shell coincide with expression (24).

\section{Conclusion}

In the present paper, we have investigated the role of the terms quadratic in the torsion fields at the quantum level in the theory with independent metric and connection fields. It turns out that:

1. In the affine metric theory the terms quadratic in the torsion fields play an auxiliary role. They serve for violating projective invariance of the action.

2. The renormalizability of the model (3) is not affected by the presence of the projective invariance.

3. In the considered model (3) the terms quadratic in the torsion fields do not contribute to the one-loop counterterms.

Let us consider the additional conditions $(20)$ arising in the definition of the quantum field propagator. It is easy to show that 


$$
d=8\left(1-b_{1}-b_{2}\right)\left(1+2 b_{1}-b_{2}\right)
$$

The coefficients $\left(1-b_{1}-b_{2}\right)$ and $\left(1+2 b_{1}-b_{2}\right)$ are proportional to the particle masses arising in the linear field approximation [15, 32, 33]. The condition $d=0$ corresponds to the presence of massless particles in the theory. In this case, the propagator of the quantum field $\gamma_{\mu \nu}^{\sigma}$ is not defined. Hence, the appearance of new massless particles is connected with the presence of the new type symmetry in the theory. We do not known the exact transformation rule of the fields under these new symmetries [33, 34, 35]. It is known that the connection field is transformed under these symmetries. The metric field is not changed.

The theory involved is renormalizable at the one-loop level on the mass-shell. The expression $\int d^{4} x \sqrt{-g}\left(R_{\alpha \beta \mu \nu} R^{\alpha \beta \mu \nu}-4 R_{\mu \nu} R^{\mu \nu}+R^{2}\right)$ is proportional to the topological number of space-time, the so-called Euler number, defined as

$$
\chi=\frac{1}{32 \pi^{2}} \int d^{4} x \sqrt{-g}\left(R_{\alpha \beta \mu \nu} R^{\alpha \beta \mu \nu}-4 R_{\mu \nu} R^{\mu \nu}+R^{2}\right)
$$

Hence, this expression is some number. In the topological trivial space-time this number is equal to zero. Then, at the one-loop level on mass-shell one needs to renormalize only the cosmological constant. Let us represent the cosmological constant in the following form:

$$
\Lambda=\frac{\lambda}{k^{2}}
$$

where $\lambda$ is the dimensionless constant. Then, from the explicit calculations in the previous section, we get the renormalization group equation

$$
\beta(\bar{\lambda})=\mu^{2} \frac{\partial \bar{\lambda}}{\partial \mu^{2}}=-\frac{29}{160 \pi^{2}} \bar{\lambda}^{2}
$$

where $\mu^{2}$ is the renormalization point mass. Hence, we have the asymptotic freedom for $\lambda$.

The result of the one-loop calculations on mass-shell coincides with the one-loop counterterms of the Einstein gravity with the cosmological constant [36]. This coincidence is accidental. The considered theory coincides with the Einstein gravity at the tree level. In the nonrenormalizable theories the results of the loop calculations depend on the choice of the dynamical variables. It has been shown [37] that the classical theory written in a different way leads to inequivalent quantum results. Since the Einstein gravity and theory under consideration are not renormalizable at the two-loop level, the equivalence of the above-mentioned theories can be violated at the quantum level. Therefore, one cannot predict the result of the one-loop calculations (24) without the corresponding calculations.

We are greatly indebted to L.O.Vasilyeva, A.Gladyshev and G. Sandukovskaya for critical reading of the manuscript. 


\section{References}

[1] G. t'Hooft and M. Veltman (1974) Ann. Inst. H. Poincarè A20, 69

[2] M. H. Goroff and A. Sagnotti (1986) Nucl. Phys. B266, 709

[3] A. E. M. van de Ven (1992) Nucl. Phys. B378, 309

[4] K. S. Stelle (1977) Phys. Rev. D16, 953

[5] I. G. Avramidi and A. O. Barvinsky (1985) Phys. Lett. B159, 269

[6] I. Antoniadis and E. T. Tomboulis (1986) Phys. Rev. D33, 2756

[7] D. A. Johnston (1988) Nucl. Phys. B297, 721

[8] F. W. Hehl, P. van der Heyde, G. D. Kerlick and I. M. Nester (1976) Rev. Mod. Phys. 48, 393

[9] D. D. Ivanenko, P. I. Pronin and G. A. Sardanashvily (1985) Gauge theory of Gravity (University Publ. House, Moscow )

[10] E. W. Mielke (1987) Geometrodynamics of Gauge Fields (Akademie-Verlag, Berlin)

[11] F. W. Hehl (1985) Found. Phys. 15, 451

[12] A. A. Tseytlin (1982) Phys. Rev. D26, 3327

[13] E. A. Lord (1978) Phys. Lett. A65, 1

[14] Y. Ne'emann and D. Šijački (1988) Phys. Lett. B200, 489

[15] K. Hayashi and T. Shirafuji (1980) Prog. Theor. Phys. 64, 866, 883, 1435, 2222; (1981) ibid. 65, 525

[16] F. W. Hehl, G. D. Kerlic and P. von der Heyde (1976) Phys. Lett. B63, 446

[17] F. W. Hehl, E. A. Lord and Y. Ne'emann (1977) Phys. Lett. B71, 432; (1978) Phys. Rev. D17, 428

[18] F. W. Hehl, J. D. McCrea, E. W. Mielke and Y. Ne'emann (1989) Found. Phys. 19, 1075

[19] E. W. Mielke, J. D. McCrea, Y. Ne'emann anf F. W. Hehl (1993) Phys. Rev. D48, 673

[20] M. Martellini (1983) Phys. Rev. Lett. 51, 152

[21] J. Dell, J. L. de Lyra and L. Smolin (1986) Phys. Rev. D34, 3012

[22] Buchbinder I. L. and Shapiro I. L. (1984) Sov. Phys. J. 27, 677 
[23] C. Y. Lee and Y. Ne'emann (1990) Phys. Lett. B242, 59

[24] M. Yu. Kalmykov and P. I. Pronin (1991) Nuovo Cimento B106, 1401

[25] V. D. Sandberg (1975) Phys. Rev. D12, 3013

[26] F. W. Hehl, W. Kopchynski, I. D. McCrea and E. W. Mielke (1991) J. Math. Phys. 32, 2169

[27] M. Yu. Kalmykov, P. I. Pronin and K. V. Stepanyantz (1994) Class. Quantum Grav. 11, 2645

[28] B. S. DeWitt (1967) Phys. Rev. 162, 1195, 1239

[29] B. S. DeWitt (1965) Dynamical Theory Groups and Fields (Gordon and Breach, New York)

[30] P. B. Gilkey (1975) J. Differ. Geomet. 10, 601

[31] A. O. Barvinsky and G. A. Vilkovisky (1985) Phys. Rep.119, 1

[32] M. Blagojevic and I. A. Nikolic (1983) Nuovo Cimento B73, 258

[33] M. Blagojevic and M. Vasilic (1986) Phys. Rev. D34, 357

[34] I. Bars and S. W. MacDowell (1983) Phys. Lett. B129, 182

[35] K. Sundermeyer (1984) Phys. Lett. B134, 415

[36] S. M. Christensen and J. Duff (1980) Nucl. Phys. B170 [FS1], 480

[37] M. Yu. Kalmykov and P. I. Pronin, Ambiguity of the one-loop calculations in a nonrenormalizable quantum gravity, (hep-th/9503215) 